\title{
Population viability analysis
}

\author{
Michael A. McCarthy
}

The University of Melbourne, Australia

Hugh P. Possingham

The University of Queensland, Australia

Keywords: biodiversity; extinction; population dynamics; risk; stochasticity; uncertainty

\begin{abstract}
Population viability analysis (PVA) assesses risks of population decline of species, and how those risks can be managed. Typically, stochastic population models are used to characterize the deterministic and stochastic components that govern changes in population size. Sources of stochasticity include demographic stochasticity due to the chance birth and death of individuals, stochasticity in the environment that is common to all individuals, chance variation in genetic composition, and spatial structure. These factors can interact to create extinction vortices that drive population decline. Predicted risks of population decline from PVA models are usually uncertain due to imprecise parameter estimates, imperfect knowledge about factors that influence dynamics, and uncertainty about future conditions. Despite these sources of uncertainty, changes in risks due to management can be predicted more reliably. Using these changes in risk, PVA can
\end{abstract}


help determine efficient management strategies for reducing high contemporary rates of extinction.

The process of using models to determine risks of decline faced by populations was initially defined as population vulnerability analysis [1], but is now known as population viability analysis (PVA). It originated from legislative requirements to assess the extinction probability of species [2], but now incorporates assessment of how risks of population decline can be reduced through appropriate management. In the face of seemingly very high contemporary rates of extinction [3], it is important that limited budgets are spent on the most efficient management strategies [4]. Using both well-established and contemporary numerical and analytical methods to build models of population dynamics that use available data and expert opinion, PVA can be a valuable tool to identify such strategies [5].

Classical PVA models typically describe how the population growth rate, or its components of fecundity and survival, change with population size or aspects of the environment (e.g., the abundance of food resources or possibly predators). Such models are typically stochastic, capturing the common unpredictability of population growth and decline. Random factors and imperfect knowledge of biological processes cause uncertainty in population forecasting. Factors that contribute to uncertainty about future population sizes include demographic stochasticity, environmental stochasticity, genetic stochasticity, and spatial variation [2].

Demographic stochasticity arises because populations are composed of individuals of different ages and sexes and their reproduction and survival are probabilistic [6]. Interactions between demographic events can amplify the variation beyond what would be expected from independent 
events. For example, deaths might precipitate other deaths due to parental relationships, or births might occur in clutches [7]. However, variation in abundance due to demographic stochasticity declines with increasing population size, and is usually unimportant for population sizes above a few hundred individuals. However, the importance of demographic stochasticity depends on the details of the model, including differences between individuals and the source of those differences $[7,8]$.

Environmental stochasticity results from unpredictable changes in the environment that affect all members of the population. For example, fluctuations in the abundance of habitat elements such as food, predators, and nest sites cause birth and death rates to change over time. Because the effect of environmental fluctuations on population dynamics may be independent of population size, environmental stochasticity may be important for quite large populations, depending on the level of variation. Although they are often considered separately, catastrophes are extreme forms of environmental stochasticity, in which random events cause large changes in the population dynamics [9].

Genetic stochasticity refers to random differences and changes in the genetic make-up of individuals and populations that may lead to changes in survival and fecundity rates [2]. Loss of genetic diversity may limit adaptation of species to changing environments, and it may also lead to inbreeding depression, in which the survival and/or reproduction of inbred individuals is reduced. The variance in the frequency of genes passed on each generation increases as population size declines, resulting in a greater risk that random drift will lead to a loss of genetic variability. 
Spatial models of PVA can integrate multiple models of local population dynamics by specifying correlations and dispersal rates among them [10]. Such a collection of populations is known as a metapopulation. Rather than modelling the local population dynamics, an alternative is to reduce the local population dynamics to simple predictions of extinction and colonization that ignore population size. The state of these stochastic patch occupancy metapopulations is measured by the number (and perhaps identity) of occupied patches [11].

Spatial structuring of populations also contributes to variation in abundance [12]. It is difficult to make general conclusions about the effect of spatial structure on meta-population dynamics, however, some frameworks exist $[13,14]$. As population fragmentation increases, the size of individual populations may decline, leading to higher levels of demographic stochasticity and often reducing the chance of dispersal between populations. Although these factors may increase the risk of local extinctions, separation may decrease correlation in environmental variation among populations. This will decrease the correlation between local extinction events and provide opportunities for recolonisation of declining or extinct populations. Risks of metapopulation extinction will depend on the combination of migration rates among populations, the level of demographic and environmental stochasticity, and the environmental correlation among populations $[15,16]$. Furthermore, dispersal characteristics within and between populations will influence the chance of breeding between related individuals, and therefore may affect genetic stochasticity.

The stochastic factors that influence the persistence of a population interact. Fluctuations resulting from environmental stochasticity may decrease population size, which in turn may exacerbate the impact of demographic stochasticity and inbreeding. Therefore, these stochastic processes are linked by feedback mechanisms, and may be thought of as "vortices" that draw a 
population closer to extinction [1]. PVA models attempt to synthesize the array of stochastic and deterministic processes that might affect the persistence of the populations being modeled.

Aside from this classical approach to PVA, predictions of extinction risk can be derived using

analysis of time series data on population sizes coupled with diffusion equation approximations

$[17,18,19]$. Further, we can infer the viability of populations by looking at species living on islands and how long they may have persisted there.

\section{Measures of threat}

The time to extinction is a readily interpretable measure of threat. However, because of sources of uncertainty described above, it is impossible to predict precisely the time to extinction. Instead, the time to extinction may be expressed as a probability distribution $[2,20]$. These distributions are typically summarized by calculating the mean time to extinction, which will overestimate the likely time to extinction because the distributions are almost always skewed towards longer extinction times [20].

A particularly relevant index of population vulnerability is the probability of extinction. Quasiextinction risk is a more general term that refers to the probability that a population will decline to or below a specified threshold at least once within a given time [17].

Quasi-extinction risk curves plot quasiextinction risk versus the threshold population size (Fig. 1). The area to the left of a quasi-extinction risk curve is equal to the expected minimum population size, which usefully summarizes the curve [21]. Therefore, a change in the expected minimum population size due to management measures the area between two quasiextinction risk curves. 


\section{Linking theory to application}

PVA has found application in many areas of conservation: determining the minimum viable population size (MVP), setting targets for conservation planning, classifying species for the International Union for Conservation of Nature (IUCN) Red List and choosing between management options for securing or recovering a threatened species. Computer programs used for PVA analysis can be case specific [15, 22], or based on generic software (e.g., RAMAS: http://www.ramas.com/software.htm; VORTEX: http://www.vortex9.org/vortex.html).

Gilpin and Soulé [1] viewed PVA as a method of determining the MVP of a species, i.e., the population size above which the risk of extinction within a specified time period is acceptably small. Such a definition implies that there is some critical population threshold below which the risk of extinction increases rapidly.

The MVP concept can be misleading in several respects. Firstly, the risk of extinction changes over a gradual continuum. Populations larger than the MVP are not safe. Populations smaller than the MVP are not sure to become extinct. Further, the MVP produced by the analysis might be very misleading. Population models are used to predict risks of extinction over considerable periods, but available data usually span a few years at the most. Such long-term predictions are subject to substantial error [19], especially when important aspects of the population dynamics are unknown. Given that the numbers produced in MVP analyses are subject to substantial error, it seems unwise to use these numbers to define "safe" population sizes.

Conservation planning demands that systems of protected areas "adequately" represent populations of all species. This invariably involves setting areal targets for a suite of well-known taxa, usually vertebrates, that need to be met for a reserve system to be viable [23]. While this is 
an appealing notion, it relies on the notion of an MVP, and hence is often not practical. However, conservation plans that seek to maximize the chance of achieving an acceptable outcome might be subject to less uncertainty [24].

The IUCN have a systematic approach of classifying threatened species [25]. This uses rules about population decline, size and extent to place every species in one of several categories such as endangered or vulnerable. The rules are intended to reflect the outcomes of PVA, and one of the criteria for classification is a credible PVA model, something which is not possible for most species due to data and time constraints.

Imprecision in PVA predictions has led to questions about its value [19]. However, requirements to predict risk still exist even if the predictions are uncertain. PVA is a formal method for synthesizing data and other sources of information for prediction. It is hard to see how any other method would provide more reliable results [25]. Further, while the actual predictions of PVA can be uncertain $[19,26]$, changes in the level of threat faced by a species can be reliable [5]. This highlights one of the most valuable roles of PVA, which is to help identify efficient management strategies through careful evaluation of existing data [5, 27, 28].

Even in the presence of uncertainty about the best management strategy, PVA can be used to identify the aspects of the model that have the greatest influence on the uncertainties through sensitivity analysis [29]. This would help managers determine the aspects of the population dynamics that are most importantly resolved in order to obtain more accurate predictions. A subtle but important difference is that PVA can also be used to determine the aspects of the population dynamics that should be resolved to help choose between the possible management strategies [30]. The difference here is that some uncertainties that cloud the fate of the population 
might not obscure the choice between different management strategies. Thus, PVA can be used as a basis for adaptive management [31].

The best stochastic population models are those that attempt to integrate the available information. It is unrealistic to assume that the long-term predictions of these models are correct, especially when they are based on only a small amount of data. The benefit of PVA is that it helps to ensure rigorous thinking about the dynamics of the population being modeled. It is important to view population models as a synthesis of information that is always incomplete and might be misleading [32].

Risks of population decline are useful and readily interpretable measures of threat in environmental management, and allow impacts to be expressed in terms of change in risks. PVA provides a framework for assessing these risks and choosing the best course of action.

\section{Acknowledgements}

This publication was supported by The Australian Research Council Centre of Excellence for Environmental Decisions.

\section{References}

[1] Gilpin, M.E. \& Soulé, M.E. (1986). Minimum viable populations: the processes of species extinction, in Conservation Biology: the Science of Scarcity and Diversity, M.E. Soulé, ed., Sinauer, Sunderland, Massachusetts, pp. 19-34.

[2] Shaffer, M.L. (1981). Minimum population sizes for species conservation. BioScience 31, 131-134. 
[3] Pimm, S.L., Russell, G.J.; Gittleman, J.L. \& Brooks, T.M. (1995). The future of biodiversity. Science 269, 347-350.

[4] Joseph, L.N., Maloney, R.F. \& Possingham, H.P. (2009). Optimal allocation of resources among threatened species: a project prioritization protocol. Conservation Biology 23, 328-338.

[5] McCarthy, M.A., Andelman, S.J. \& Possingham, H.P. (2003). Reliability of relative predictions in population viability analysis. Conservation Biology 17, 982-989.

[6] Kendall, D.G. (1948). On the generalised birth and death process. Annals of Mathematical Statistics 19, 1-15.

[7] McCarthy, M.A., Franklin, D.C. \& Burgman, M.A. (1994). The importance of demographic uncertainty: an example from the helmeted honeyeater. Biological Conservation 67, 135-142.

[8] Melbourne, B.A. \& Hastings, A. (2008). Extinction risk depends strongly on factors contributing to stochasticity. Nature 454, 100-103.

[9] Caughley, G. (1994). Directions in conservation biology. Journal of Animal Ecology 63, 215244.

[10] Hanski , I.A. \& Gilpin, M.E. (1997). Metapopulation Biology: Ecology, Genetics, and Evolution. Academic Press, San Diego.

[11] Day J.R. \& Possingham H.P. (1995). A stochastic metapopulation model with variability in patch size and position. Theoretical Population Biology 48, 333-360.

[12] Gilpin, M.E. (1987). Spatial structure and population vulnerability, in Viable Populations for Conservation, M.E. Soulé, ed., Cambridge University Press, Cambridge, pp. 125-139. 
[13] Ovaskainen, O. \& Hanski, I. (2001). Spatially structured metapopulation models: global and local assessment of metapopulation capacity. Theoretical Population Biology 60, 281-302.

[14] Drechsler, M. \& Johst, K.(2010). Rapid viability analysis for metapopulations in dynamic habitat networks. Proceedings of the Royal Society B-Biological Sciences 277, 1889-1897.

[15] McCarthy, M.A. \& Lindenmayer, D.B. (2000). Spatially-correlated extinction in a metapopulation of Leadbeater's possum. Biodiversity and Conservation 9, 47-63.

[16] Elkin C.M. \& Possingham H. (2008). The role of landscape-dependent disturbance and dispersal in metapopulation persistence. American Naturalist 172, 563-575.

[17] Ginzburg, L.R., Slobodkin, L.B., Johnson, K. \& Bindman, A.G. (1982). Quasiextinction probabilities as a measure of impact on population growth. Risk Analysis 2, 171-81.

[18] Lande, R. (1993). Risks of population extinction from demographic and environmental stochasticity and random catastrophes. American Naturalist 142, 911-927.

[19] Fieberg, J. \& Ellner, S.P. (2000). When is it meaningful to estimate an extinction probability? Ecology 81, 2040-2047.

[20] Mangel, M. \& Tier, C. (1994). Four facts every conservation biologist should know about persistence. Ecology 75, 607-614.

[21] McCarthy, M.A. \& Thompson, C. (2001). Expected minimum population size as a measure of threat. Animal Conservation 4, 351-355. 
[22] McCarthy, M.A. (1996). Red kangaroo (Macropus rufus) dynamics: effects of rainfall, harvesting, density dependence and environmental stochasticity. Journal of Applied Ecology 33, $45-53$.

[23] Moilanen A., Wilson, K.A. \& Possingham, H. (eds) (2009). Spatial Conservation Prioritization. Oxford University Press, London.

[24] McCarthy, M.A., Thompson, C.J., Moore, A.L. \& Possingham, H.P. (2011). Designing nature reserves in the face of uncertainty. Ecology Letters 14, 470-475.

[25] IUCN (2001). IUCN Red List Categories and Criteria, Version 3.1. IUCN Species Survival Commission, Gland, Switzerland.

[25] McCarthy, M.A., Keith, D., Tietjen, J., Burgman, M.A., Maunder, M., Master, L., Brook, B.W., Mace, G., Possingham, H.P., Medellin, R., Andelman, S., Regan, H., Regan, T. \& Ruckelshaus, M. (2004). Comparing predictions of extinction risk using models and subjective judgement. Acta Oecologica 26, 67-74.

[26] Lindenmayer, D.B., Possingham, H.P., Lacy, R.C., McCarthy, M.A. \& Pope, M.L. (2003). How accurate are population models? Lessons from landscape-scale tests in a fragmented system. Ecology Letters 6, 41-47.

[27] Possingham, H.P., Lindenmayer, D.B. \& Norton, T.W. (1993). A framework for the improved management of threatened species based on PVA. Pacific Conservation Biology 1, 3945. 
[28] Lindenmayer, D.B. \& Possingham, H.P. (1996). Ranking conservation and timber management options for Leadbeater's Possum in southeastern Australia using Population Viability Analysis. Conservation Biology 10, 235-251.

[29] McCarthy, M.A., Burgman, M.A. \& Ferson, S. (1995). Sensitivity analysis for models of population viability. Biological Conservation 73, 93-100.

[30] Baxter, P.W.J., McCarthy, M.A., Possingham, H.P., Menkhorst, P. \& McLean, N. (2006). Accounting for management costs in sensitivity analyses of matrix population models. Conservation Biology 20, 893-905.

[31] Bakker,V.J. \& Doak D.F. (2009). Population viability management: ecological standards to guide adaptive management for rare species. Frontiers in Ecology and the Environment 7, 158165.

[32] Burgman, M.A. \& Possingham, H.P. (2000). Population viability analysis for conservation: the good, the bad and the undescribed, in Genetics, Demography, and Viability of Fragmented Populations, A.G. Young \& G.M. Clarke, eds, Cambridge University Press, Cambridge, pp. 97112. 


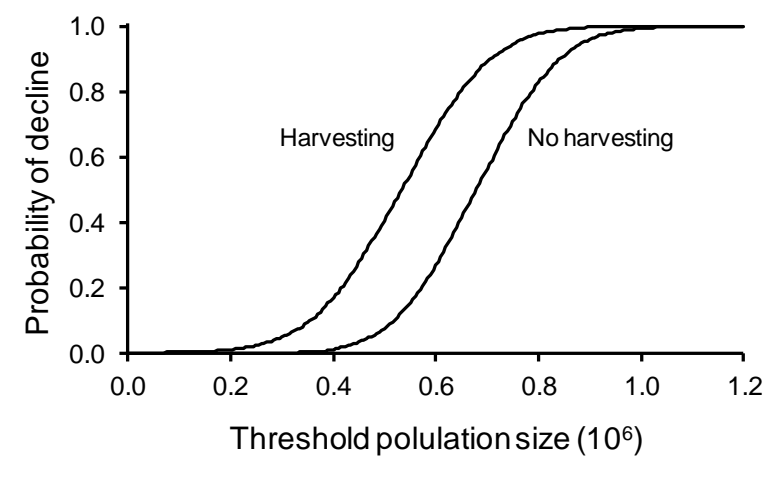

Fig. 1. Quasi-extinction risk curves for a modelled red kangaroo population subject to harvesting of 100,000 individuals per year, and in the absence of harvesting [22]. The quasi-extinction risk curve plots the probability of the population falling below the threshold population size at least once within the next 20 years. The area between the curves (148269) is the change in the expected minimum population size due to harvesting, measuring how much closer the population is to extinction. 